\title{
Vital Dyes in Vitreomacular Surgery
}

Authors: Peter Bracha $\mathrm{MD}^{1}$, Tom A. Ciulla $\mathrm{MD}^{1,2}$, Caroline R. Baumal MD ${ }^{3}$

1. Department of Ophthalmology, Indiana University School of Medicine, Indianapolis, Indiana, USA

2. Retina Service, Midwest Eye Institute, Indianapolis, Indiana, USA

3. Tufts University School of Medicine, New England Eye Center, Boston, Massachusetts, USA

Corresponding author:

Peter Bracha

Glick Eye Institute

1160 West Michigan Street

Indianapolis, IN

46202

pbracha@iu.edu

Grant support or research funding: None

Proprietary interests in material described: None

This material was not presented at any scientific meeting.

Disclosures:

P Bracha has no financial interest in the subject matter of this review

$\mathrm{T}$ Ciulla has no financial interest in the subject matter of this review. He has an employment relationship with Spark Therapeutics; however, this manuscript was written during his work as a Volunteer Clinical Professor of Ophthalmology at Indiana University School of Medicine, and none of the work herein represents any official position or opinion of Spark Therapeutics or its management.

C. Baumal has no financial interest in the subject matter of this review. She has served as a consultant for Stealth Biotherapeutics, advisory Board for Genentech, speaker for Optovue and Zeiss.

Key words: Chromovitrectomy, vital dye, internal limiting membrane, epiretinal membrane, indocyanine green, brilliant blue $\mathrm{G}$, trypan blue, triamcinolone acetonide

This is the author's manuscript of the article published in final edited form as:

Bracha, P., Ciulla, T. A., \& Baumal, C. R. (2018). Vital Dyes in Vitreomacular Surgery. Ophthalmic Surgery, Lasers \& 
Abstract: Vital dyes contain complex molecules with chromophores that stain living tissues and have greatly enhanced identification and removal of transparent vitreoretinal tissues during surgery. Several "chromovitrectomy" dyes are frequently used by vitreoretinal specialists, including indocyanine green, trypan blue, brilliant blue $\mathrm{G}$ and triamcinolone acetonide; other dyes are also under investigation. Currently available chromovitrectomy dyes have their limitations, and of particular concern is their possibility for acute and chronic toxicity to the neurosensory retina and retinal pigmented epithelium. The potentially irreversible acute toxicity and other limitations, such as lack of long-term safety profiles, highlight the need for a review of the current literature and for further advancements. 


\section{Introduction}

In all surgical fields, visualization of tissues and anatomic planes assumes paramount importance. In vitreoretinal surgery, the surgical planes may be only microns thick, and neighbouring nerve fiber layer tissues are susceptible to mechanical damage with secondary adverse visual sequelae. Thus, surgical dissection in the appropriate plane without traumatizing underlying retinal structures is critical. Particularly challenging in retinal surgery is the visualization of transparent preretinal tissues such as internal limiting membrane (ILM), epiretinal membrane (ERM) and the vitreous cortex. Vital dyes are complex molecules containing chromophores, the structure of a

molecule responsible color, ${ }^{1}$ that stain living tissues and have greatly enhanced identification and removal of transparent anatomical layers during vitreoretinal surgery.

Currently available chromovitrectomy dyes do have their limitations. The properties of an ideal dye for vitreoretinal surgery include lack of toxicity to all retinal layers, easy application and extraction from the eye, excellent staining and contrast of desired tissues, no potential for phototoxicity, low cost, minimal preparation and existing Food and Drug Administration (FDA) approval. Several chromovitrectomy dyes are frequently used by vitreoretinal specialists, including indocyanine green (ICG), trypan blue (TB), brilliant blue $\mathrm{G}$ (BBG) and triamcinolone acetonide (TA); other dyes are also currently under investigation. The 2017 American Society of Retinal Specialists (ASRS) Preferences and Trends (PAT) survey demonstrated that of United States (US) retina specialists survey responders, $69.0 \%$ percent preferred to use ICG to aid in ERM and/or ILM peeling, 9.5\% preferred TA, $14.8 \%$ used BBG, $1.2 \%$ used TB, 3.1\% preferred no dye, and $2.4 \%$ used an unlisted dye. ${ }^{2}$ Additionally, the last decade has seen a trend towards more US providers utilizing ICG, despite some reports of toxicity, with a slight decrease in the use of TA and TB (Figure 1). ${ }^{2-6}$ 
Of particular concern with chromovitrectomy dyes is the potential for acute and/or chronic toxicity to the neurosensory retina and retinal pigmented epithelium (RPE), and the toxicity of commonly used dyes is discussed throughout this text. While most physicians peel the ILM during macular hole surgery due to the improvement in macular hole closure rates, the visual results of ILM peeling during ERM resection are less convincing. Despite reducing the rate of recurrence of ERMs, ILM peeling has not convincingly improved visual outcomes, and the recurrence rate of ERMs is already relatively low. ${ }^{7}$ The potential for harmful effects, varied practice patterns and other limitations highlight the need for a current literature review and further advancements.

\section{Historical perspective: Introduction of ICG-assisted ILM peeling}

Surgical repair of idiopathic macular holes has undergone significant evolution in the last three decades. In 1991, Kelly and Wendel published a technique for surgical closure, in which pars plana vitrectomy (PPV) was combined with intraocular gas tamponade and face down positioning. This procedure resulted in 58\% macular hole closure rate and $42 \%$ improvement in visual acuity (VA), a tremendous improvement compared to observation. ${ }^{8}$ Over the ensuing decade, various modifications to this technique were investigated, with the goal of improving closure rates and visual outcomes. The technique that is currently popular involves removal of the ILM around the macular hole. It is hypothesized that ILM removal reduces tangential forces on the fovea, which act as a potential mechanism for failure of macular hole closure. In 1997, Eckardt et al. published a series of 39 full-thickness macular holes that underwent ILM peeling during PPV, and demonstrated a notable improvement in closure rate of $92 \%{ }^{9}$ Follow-up studies confirmed these closure rates, effectively establishing the role of ILM peeling in macular hole repair. By 2014, the ASRS PAT survey noted $93 \%$ of 
responding US retinal physicians performed routine ILM peeling during macular hole surgery. 5

One of the technical difficulties in peeling the ILM is adequate visualization of this thin transparent membrane, with concern for damage to the inner retinal nerve fiber layer and permanent scotoma from excessive manipulation. Challenges in consistent visualization of ILM led investigators to evaluate ICG as a dye to stain this transparent layer. ICG is a water-soluble, tricarbocyanine dye with infrared absorption properties that was initially used as a contrast agent in radiology. Prior to its use for ILM peeling, ICG has been used for imaging of the choroidal circulation and was also investigated for staining of the anterior lens capsule in cataract surgery. ${ }^{10} \mathrm{~A}$ favorable safety profile for ICG was suggested by the lack of adverse effects when it was used for dye-based angiography, even in cases where large amounts of ICG dye leaked into or under the retina. ${ }^{11,12}$ For surgical use, ICG in the form of a powder must be dissolved in sterile water and diluted to the desired concentration.

In 2000-2001, teams in both Japan and the US evaluated ICG-assisted ILM peeling in animal models, and the preliminary safety and success of these preclinical studies led to human trials. In humans, following PPV and PVD induction, the ICG was applied directly to the ILM for several minutes and was then aspirated. The staining provided enhanced visualization of the ILM and aided in its peeling. The resulting supportive publications with excellent images, along with the conceptual appeal, good outcomes and early lack of adverse events generated positive response within the vitreoretinal community. ${ }^{13,14}$

Over the ensuing years, various groups replicated the successful staining demonstrated in these original studies. ${ }^{15-17}$ However, case reports of potential toxicity related to ICG soon surfaced, including reports of worse visual acuity outcomes and 
peripheral visual field defects. ${ }^{18-22}$ Retinal pigmentary changes were observed in up to $50 \%$ in some series and reports of RPE atrophy surfaced as well. ${ }^{23-25}$ However, the development of RPE changes following PPV may be unrelated to the use of ICG, as the Vitrectomy for Macular Hole Study Group in 1997 was performed prior to the application of ICG or other dyes, and noted that $33 \%$ of patients developed RPE changes. ${ }^{26}$ In 2001, Gandorfer et al. worked on elucidating the potential reasons for these adverse outcomes by performing electron microscopy on excised ILMs. To their surprise, microscopy revealed that, in addition to ILM removal, plasma membranes of Muller cells, Muller cell foot plates and other undetermined cellular debris were excised. ${ }^{18}$ In contrast, ILM peeling without the assistance of ICG, did not reveal excessive excision of non-ILM tissue. ${ }^{27}$

While the exact mechanisms for ICG-related injury to retinal tissue remains controversial, studies suggest that ICG has direct toxicity to the RPE, especially if utilized at higher concentrations and over longer periods ofcontact during surgery. The hypothetical mechanisms for ICG-related RPE and neurosensory retinal damage are summarized in Table 1, along with suggested techniques to minimize damage.

Conflicting rates of these adverse outcomes have been published in larger studies; ${ }^{22,28-31}$ variations in ICG concentration, addition of dextrose $5 \%$ in water (D5W) to the diluent, ICG exposure time, endoillumination time, surgeon experience and the degree of irrigation confound comparison of different studies. Possibly the best evidence to date is a 2012 meta-analysis of both retrospective and randomized, prospective studies. This analysis compiled the results from twenty-two studies and 1585 eyes, and concluded that ICG-assisted ILM peeling was associated with increased rates of visual field defects and worse visual acuity outcomes compared to ILM peeling without ICG. In particular, the percentage of patients who improved to better than 
20/40 was lower in those with the ICG-assisted peeling compared to the those without ICG (OR $0.61,95 \%$ CI 0.43 to $0.97, \mathrm{p}=0.033$ ), despite similar macular hole closure rates. ${ }^{32}$ Based on the potential adverse effects of ICG, there continues a search for alternative dyes for routine staining of the ILM. Alternatively, steps should be taken to minimize the concentration, exposure time and illumination during surgery.

Table 1. Hypothetical mechanisms for indocyanine green (ICG)-related tissue damage and suggested techniques to minimize this damage.

\begin{tabular}{|c|c|}
\hline $\begin{array}{l}\text { Hypothetical mechanisms } \\
\text { for ICG-related tissue } \\
\text { damage }\end{array}$ & Suggested techniques to minimize damage \\
\hline $\begin{array}{l}\text { Dose- and time-dependent } \\
\text { direct toxicity to RPE }\end{array}$ & $\begin{array}{l}\text { Minimize the dose }(0.5 \mathrm{mg} / \mathrm{mL}) \text { and minimize exposure time during } \\
\text { surgery. }{ }^{35} \text { Copiously irrigate ICG to minimize chronic direct } \\
\text { toxicity. Avoid direct injection to the macular hole or use various } \\
\text { materials (ex: viscoelastic material) to create a barrier between ICG } \\
\text { and RPE. }\end{array}$ \\
\hline $\begin{array}{l}\text { Acute and chronic } \\
\text { phototoxicity }^{38}\end{array}$ & $\begin{array}{l}\text { Minimize direct and proximal light-pipe illumination during } \\
\text { surgery. }{ }^{39} \text { Copiously irrigate ICG to minimize chronic phototoxicity. } \\
\text { Dissolving the dye in BSS or D5W alters the absorption spectrum } \\
\text { and can minimize phototoxicity. }{ }^{40}\end{array}$ \\
\hline $\begin{array}{l}\text { More aggressive surgical } \\
\text { excision }^{41,42}\end{array}$ & $\begin{array}{l}\text { Minimize manipulation of ILM and underlying neurosensory retina. } \\
\text { However, the aggressive surgical excision may in part be due to ICG } \\
\text { chemically modifying the surgical plane. }\end{array}$ \\
\hline $\begin{array}{l}\text { Hypo-osmotic effects } \\
\text { (controversial) }^{33,43,44}\end{array}$ & Utilize a physiologic osmolarity \\
\hline
\end{tabular}


BSS, balanced salt solution; D5W, 5\% dextrose in water; ICG, indocyanine green; RPE, retinal pigment epithelium

This table describes the hypothetical mechanisms for ICG-related retina toxicity and techniques to minimize potential toxicity.

ICG has separately been investigated to facilitate epiretinal membranesurgery. While several studies have confirmed poor ERM staining with ICG as highlighted in Figure 2, the negative ICG staining of ERM accompanied by staining of adjacent ILM may facilitate removal of both ERM and ILM combined. ${ }^{45,46}$ Negative staining with ICG and other dyes, wherein the lack of staining of the tissue of interest, contrasted by staining of adjacent tissue, improves visualization and can aid in excision. Negative staining techniques have been applied to the removal of vitreous, posterior hyaloid, ERM and ILM. ${ }^{47}$

Other dyes such as TB and triamcinolone, discussed later in this review, more favourably stain ERMs. Finally, infracyanine green (IFCG), a biosimilar dye to ICG, was briefly investigated as an alternative to ICG and was hypothesized to have an improved safety profile due to a more physiological osmolarity as well as a lack of

iodine. ${ }^{48-52}$ After several published investigations, alternate dyes such as trypan blue and brilliant blue G gained more investigative enthusiasm and IFCG is not routinely used.

\section{Brilliant Blue $\mathbf{G}$}

Brilliant blue $\mathrm{G}$, also known as acid blue 90 and Coomassie BBG, is a blue dye that non-selectively binds to most proteins and has been utilized for protein staining in biologic fields. The potential toxicity of ICG led investigators in Japan to evaluate the 
safety of this dye. In 2006, Enaida et al. published a thorough evaluation of various doses of BBG in vitrectomized rat eyes. Following successful preclinical evaluation, BBG was investigated in humans as a dye for ERM and ILM peeling (Figure 3). In 2006, Enaida et al. published the successful application of $0.25 \mathrm{mg} / \mathrm{ml}$ of BBG during epiretinal membrane resection and ILM peeling for macular hole repair in a small group of eyes. No BBG-related adverse events were noted, but peripheral visual field evaluation was not performed. Similarly, ERMs were well-visualized and effectively peeled, without documented BBG-related adverse events. Follow-up studies confirmed efficacy of BBG in ILM staining, with minimal observed toxicity. ${ }^{53} \mathrm{~A}$ recent review and meta-analysis of 846 eyes demonstrated the superior visual outcomes of BBG compared to ICG for ILM peeling in macular hole surgery. ${ }^{54}$ Long-term follow-up, of an average of 2 years following surgery, similarly demonstrated superior visual acuity outcomes for BBG over ICG. ${ }^{55}$

Limitations of BBG include the possible subjective and objective inferiority of ILM staining compared to ICG, lack of FDA approval, minimal staining of ERMs and reports of adverse events. ${ }^{56}$ Despite its possible inferior staining, as compared to ICG, the dye's widespread use suggest adequate ability to aid in visualization of the ILM. The number of adverse events reported with BBG is fewer than with ICG. ${ }^{57}$ On examination of excised ILMs following the application of various dyes, BBG had similar findings as $\mathrm{TB}, \mathrm{BPB}$ and $\mathrm{CB}$, and specimens did not contain large cellular fragments and Muller cell end-feet as was found with ICG derived ILM specimens. ${ }^{58}$ The clinical significance of the excision of a small amount of retinal tissue still remains to be elucidated. Similar to the use of ICG, greater light intensity and duration, as well as higher concentrations of BBG and contact time, have been demonstrated in vivo to 
result in cell toxicity; thus, application dose and duration should be minimized during surgery. ${ }^{59}$

Currently, approximately $14.8 \%$ of US providers utilize BBG for aid in ILM peeling. ${ }^{2}$ For surgical use, it can be purchased as a solution that is then diluted to a desired concentration. It is commercially available as Brilliant Peel® (Fluoron, Ulm, Germany) and Ocublue Plus ${ }^{\circledR}$ (Aurolab, Madurai, India). It is available in combination with trypan blue as Bio-Blue DUO ${ }^{\circledR}$ (Biotech Visioncare, Gujarat, India) and Membrane Blue-Dual ${ }^{\circledR}$ (Dutch Ophthalmic Research Center, Zuidland, Netherlands), and with trypan blue and lutein as Doubledyne ${ }^{\circledR}$, Tripledyne ${ }^{\circledR}$ and Retidyne ${ }^{\circledR}($ Kemin Industries, Inc., U.S.A). Overall, BBG appears to have fewer safety concerns than ICG but, it has slightly inferior staining properties, is not FDA approved, requires compounding and the long-term adverse and visual field effects have not been thoroughly evaluated.

\section{Triamcinolone Acetonide}

Triamcinolone acetonide (TA) is a sterile, corticosteroid, suspension that is used in one of two forms for vitreoretinal surgery: Non-preservative free triamcinolone acetonide (non-PFTA), commercially available as Kenalog® (Bristol-Myers Squibb, $\mathrm{NJ}$ ) or preservative free triamcinolone acetonide (PFTA), commercially available as Triescence ${ }^{\circledR}$ (Alcon, Fort Worth, TX). Non-PFTA is formulated in a vehicle that contains $0.99 \%$ benzyl alcohol as a preservative. While its intraocular use is off-label, non-PFTA was the initial formulation used for intravitreal injection for macular edema and then used as an adjuvant during PPV. ${ }^{60}$ There are concerns about the preservative in non-PFTA which has produced in vitro retinal cell toxicity. Thus, PFTA was developed as an alternative, and is an FDA approved corticosteroid for intraocular use. It is 
available as a $1 \mathrm{~mL}$ vial with a concentration of $40 \mathrm{mg} / \mathrm{mL}$. Advantages of PFTA include its FDA-approved status for intraocular use (Triescence) and the lack of preservatives, while non-PFTA is more cost-effective. Currently, approximately $9.5 \%$ of US providers utilize TA for aid in peeling the ERM or ILM. ${ }^{2}$

Within vitreoretinal surgery, TA can be utilized during PPV, where it adheres to vitreous to enhance visualization. It facilitates visualization of vitreous cortex and the separation of the posterior hyaloid, tissues that can be challenging to visualize due to their transparency. Upon administration into the vitreous cavity, the compound becomes trapped within the vitreous gel, where the white steroidal crystals provide contrast with adjacent tissues. Since 2000, the use of TA for vitreous visualization rapidly gained acceptance due to this effective coating ability.. ${ }^{61,62}$

In addition to the effective staining of vitreous, TA has also been investigated for other surgical applications. The corticosteroid coats the ILM, possibly due to residual collagen fibers on the transparent tissue following hyaloid separation. ${ }^{63,64}$ As opposed to staining with dyes such as ICG, TA coats tissues allowing for visualization of peeled membranes. ${ }^{65}$ Retrospective analyses of TA application in MH repair has not shown toxicity to the retina or RPE, in contrast to the early ICG for macular hole surgery studies. ${ }^{55,66}$ Furthermore, in select studies, the visual outcomes with the use of TA were superior to the use of ICG. ${ }^{67}$ Additional applications include the coating of ILM and ERM in PVR resection. ${ }^{68}$

With regard to safety, TA use during vitrectomy has been inconsistently associated with postoperative ocular hypertension. ${ }^{66,69}$ The conflicting results are likely due to the independent ocular hypertensive effects of PPV as well as the use of gas and periocular or topical corticosteroids. ${ }^{66,67}$ The rates of ocular hypertension are significantly lower than following intravitreal depot injection because most TA is 
washed out during PPV. However despite irrigation, several studies have found residual TA on or beneath the retina. Fortunately, no local untoward effects of this residual TA have been demonstrated, likely due to its indolent nature and relatively short half-life in ocular tissue (18.6 days in nonvitrectomized eyes and 3.2 days in vitrectomized eyes), with the residual TA following vitrectomy typically absorbed by 8 weeks. ${ }^{67,70-73}$ Studies have not shown increased rates of endophthalmitis or cataract formation with the use of TA during PPV despite the cataractogenic and immunosuppressive nature of corticosteroids. $^{66}$

Overall, TA is a useful adjuvant to coat the vitreous cortex during PPV and facilitate posterior hyaloid separation. Additionally, its coating ability facilitates ERM and preretinal membrane peeling. It does not stain ILM to the extent of the other vital dyes which truly stain these tissues, and thus does not enhance visualization to the same degree.

\section{Trypan blue}

Trypan blue (TB) is an anionic, hydrophilic azo dye. After favourable safety data following its use in the anterior segment as an anterior capsular stain, it has been evaluated in vitreoretinal surgery to stain epiretinal membranes and the ILM.

Favourable preclinical safety studies led to the first investigation of $\mathrm{TB}$ as an aid to ERM peeling in proliferative vitreoretinopathy. ${ }^{74}$ Shortly thereafter, TB dye demonstrated efficacy staining the ILM during macular hole surgery. ${ }^{75}$ Numerous studies indicate that TB stains both the ERM and ILM, ${ }^{75-80}$ but other authors contend that TB does not effectively stain the ILM..$^{81,82}$

Some safety concerns have arisen although not to a similar magnitude as with ICG. Similarly to ICG studies and the natural history following macular hole surgery 
without the use of vital dyes, several publications have noted development of RPE atrophy following macular hole surgery using adjuvant TB ${ }^{83,84}$ Histopathologic studies evaluating TB-surgically removed ILM specimens show variable amounts and sizes of glial elements, although typically less fragments than with ICG. ${ }^{58,85,86}$ This variability in results may be related to differences in aggressiveness of surgical excision and lack of standardization in evaluation of surgical specimens. Regardless, the clinical relevance of these neural elements on excision remains controversial, as the effect of excising non-ILM tissue during membrane peel has not been consistently demonstrated to have functional sequelae. For example, Li et al. found that despite removal of the neural elements with membranes, patients still had appropriate visual outcomes. ${ }^{85}$

A prospective, randomized trial by Haritoglou suggested an acceptable safety profile for TB without the visual field defects, RPE changes or suboptimal visual acuity outcomes that were observed after ICG-assisted ILM peeling. ${ }^{87}$ In a direct prospective, non-randomized comparison of IFCG and TB-assisted peeling of the ILM, TB had better central visual field results. ${ }^{51}$ In contrast, a prospective, randomized study, investigating a larger number of patients, found no difference in visual field or visual acuity outcomes between ICG- and TB-assisted ILM peeling in macular holes. ${ }^{88}$ These two studies provide the best quality evidence comparing TB with other dyes. Overall, they are suggestive of good central visual acuity and peripheral visual field outcomes with the use of TB, with possible but not definitive superiority over ICG.

Trypan blue (TB) was approved by the FDA in 2009 for epiretinal membrane removal and is available as MembraneBlue $0.15 \% ®$ (Dutch Ophthalmic USA). Of note is that the TB concentration approved for vitreoretinal surgery is $0.15 \%$ and differs from that of VisionBlue $0.06 \%$ which is approved for staining of the anterior lens capsule. Despite FDA approval, only 1\% of US providers utilize TB for ERM and/or ILM 
removal as per the 2017 ASRS PAT survey. ${ }^{2}$ Despite possibly fewer adverse events compared to ICG as well as FDA approval, the lack of popularity of TB stems from inferior ILM staining compared to the alternative dyes which narrows its applications.

\section{Investigational Dyes}

Numerous dyes have been proposed as alternatives to those currently used, with the ultimate goals of minimizing toxicity and maximizing visualization at minimal cost. Since 2004, most investigational dyes haven't advanced past preclinical trials and a few human case series, including Congo Red, ${ }^{89}$ Chicago Blue, ${ }^{90-92}$ E68,,${ }^{91,93}$ Evans Blue,${ }^{89,94-}$ ${ }^{96}$ Fast Green, ${ }^{89,94-97}$ Fluorometholone acetate, ${ }^{98}$ Indigo Carmine, ${ }^{96}$ Light Green,${ }^{89}$ Methyl Violet, ${ }^{89,99,100}$ Methylene Blue, ${ }^{89,94}$ Orangell, ${ }^{99}$ Patent Blue V, ${ }^{92,101-106}$ Rhodulinblua-basic 3, ${ }^{90,99}$ Rhodamine 6G, ${ }^{90,107}$ Sudan Black, ${ }^{89}$ Toluidine Blue ${ }^{89}$ and Trisodium. $^{99}$

Other dyes have been investigated to a greater degree, either historically or currently. Bromophenol Blue underwent extensive preclinical evaluation ${ }^{95,97,108}$ and the blue dye results in moderate staining of ERM, ILM and vitreous. ${ }^{89,109}$ Despite in vitro suggestion of delayed toxicity, ${ }^{110}$ the dye has been investigated in humans without evident toxicity, ${ }^{58,111}$ and is commercially available at a concentration of $1.3 \mathrm{mg} / \mathrm{ml}$ with BBG as Brilliant Peel Dual Dye ${ }^{\circledR}$ (Fluoron, Ulm, Germany).

Infracyanine green, was a promising alternative to ICG due to a physiologic osmolarity, hypothetically less cytotoxicity due to a lack of iodine, and less phototoxicity due to a higher peak wavelength absorption spectrum. ${ }^{40}$ In vitro studies supported this hypothesis and demonstrated less cytotoxicity to cultured RPE and retinal ganglion cells in comparison to BBG, ICG And bromophenol blue. ${ }^{108}$ Animal studies ${ }^{112}$ and subsequently human trials suggested good visualization of ILM without obvious 
toxicity. ${ }^{48,50,113}$ Further investigation of this dye has been surprisingly limited, with the last publication in $2013 .{ }^{95}$ The reasons for the possible decline of investigative fervour include modifications to minimize the toxicity of ICG, the regulatory approval of other dyes and experience suggestive of better outcomes with the use of other dyes (personal communication with investigator, unpublished data).

Lutein is a yellow-orange dye that is analogous to TA in that it coats intraocular tissues, in contrast to true staining. Lutein highlights the vitreous well but only coats ILM and ERM mildly. However, when combined with other dyes it hypothetically reduces cytotoxicity. The dye appears safe in preclinical ${ }^{114,115}$ and human studies $^{116}$ and is commercially available for viretoretinal use as Retidyne ${ }^{\circledR}$, Retidyne Plus ${ }^{\circledR}$ and Vitreodyne ${ }^{\circledR}$ (Kemin Industries, Inc., U.S.A).

Acai fruit is the most promising of a group of naturally occurring dyes that have recently been investigated. ${ }^{117,118}$ It is a purple-colored anthocyanin dye that preferentially stains the ILM and recently underwent human trial (Clinicaltrials.gov: NCT02691429) with the results yet to be published.

The introduction of Acid Violet to the market provides a cautionary tale. Preclinical evaluation suggested lack of toxicity at concentrations up to $0.125 \mathrm{mg} / \mathrm{ml}$, 119,120 and some concern for phototoxicity. ${ }^{92}$ Ala Medics introduced Ala Purple to the market at a concentration of $1.5 \mathrm{mg} / \mathrm{ml}$. Shortly thereafter, several publications suggesting the dye not be used due to the toxicity at commercially available concentrations, ${ }^{121-123}$ and the company pulled the product from the market. Subsequent in vitro studies further supported the toxicity profile of Acid Violet. ${ }^{124}$

If dyes pass preclinical evaluation, careful evaluation in humans is required with focus on visual fields, retinal nerve fiber layer evaluation in addition to the traditional structural OCT analysis of the vitreomacular interface. For broad acceptance and 
application, novel dyes have a major hurdle to demonstrate an interval improvement in staining and safety over those currently available. However, if less expensive to produce and distribute, they may only need to demonstrate similar safety and efficacy to become established in the market. A paucity of studies exists directly comparing commonly used dyes or comparing to ELM removal or macular hole surgery without the use of dye. Factors to evaluate include long term visual field and OCT retinal nerve fiber layer outcomes, and standard outcomes including macular hole closure rate, epiretinal membrane recurrence, visual acuity, macular OCT changes and development of RPE atrophy.

\section{Conclusion}

Vital dyes are used to facilitate excision of transparent intraocular tissues. A comparison of commonly used dyes is presented in Table 2. TA is an excellent option to stain residual vitreous and the posterior hyaloid. TA can delineate ERM and facilitate removal. However, it is inferior to stain ILM compared to other options. ICG is broadly used in vitreoretinal surgery, but concerns exist about ICG toxicity to the RPE and neurosensory retina with potential visual sequelae. It is unclear whether techniques to minimize ICG exposure, including limiting duration of contact, minimizing concentration, utilizing D5W as a solvent, avoidance of application to the macular hole and thorough irrigation following application are able to completely eliminate potential for toxicity . New dyes such as BBG and novel dyes under development, may ultimately prove superior with regards to efficacy and safety. 


\section{References}

1. IUPAC. Compendium of Chemical Terminology, 2nd ed. (the "Gold Book"). Oxford: Blackwell Scientific Publications; 1997.

2. Stone T, ed. ASRS 2017 Preferences and Trends Membership Survey: Chicago, IL. American Society of Retina Specialists. 2017.

3. Jumper J, Mittra R, eds. ASRS 2012 Preferences and Trends Membership Survey: Chicago, IL. American Society of Retina Specialists. 2012.

4. $\quad$ Mittra R, Pollack J, eds. ASRS 2009 Preferences and Trends Membership Survey: Chico, CA. American Society of Retina Specialists. 2009.

5. Stone T, ed. ASRS 2014 Preferences and Trends Membership Survey: Chicago, IL. American Society of Retina Specialists. 2014.

6. Mittra R, Jumper J, eds. ASRS 2010 Preferences and Trends Membership Survey: Chicago, IL. American Society of Retina Specialists. 2010.

7. Azuma K, Ueta T, Eguchi S, Aihara M. EFFECTS OF INTERNAL LIMITING MEMBRANE PEELING COMBINED WITH REMOVAL OF IDIOPATHIC EPIRETINAL MEMBRANE: A Systematic Review of Literature and MetaAnalysis. Retina (Philadelphia, Pa). 2017;37(10):1813-1819.

8. Kelly NE, Wendel RT. Vitreous surgery for idiopathic macular holes. Results of a pilot study. Archives of ophthalmology (Chicago, Ill : 1960). 1991;109(5):654659.

9. Eckardt C, Eckardt U, Groos S, Luciano L, Reale E. [Removal of the internal limiting membrane in macular holes. Clinical and morphological findings]. Der Ophthalmologe : Zeitschrift der Deutschen Ophthalmologischen Gesellschaft. 1997;94(8):545-551.

10. Pandey SK, Werner L, Escobar-Gomez M, Roig-Melo EA, Apple DJ. Dyeenhanced cataract surgery. Part 1: anterior capsule staining for capsulorhexis in advanced/white cataract. Journal of cataract and refractive surgery. 2000;26(7):1052-1059.

11. Hope-Ross M, Yannuzzi LA, Gragoudas ES, et al. Adverse reactions due to indocyanine green. Ophthalmology. 1994;101(3):529-533.

12. Obana A, Miki T, Hayashi K, et al. Survey of complications of indocyanine green angiography in Japan. American journal of ophthalmology. 1994;118(6):749-753.

13. Kadonosono K, Itoh N, Uchio E, Nakamura S, Ohno S. Staining of internal limiting membrane in macular hole surgery. Archives of ophthalmology (Chicago, Ill : 1960). 2000;118(8):1116-1118.

14. Da Mata AP, Burk SE, Riemann CD, et al. Indocyanine green-assisted peeling of the retinal internal limiting membrane during vitrectomy surgery for macular hole repair. Ophthalmology. 2001;108(7):1187-1192.

15. Kwok AK, Li WW, Pang CP, et al. Indocyanine green staining and removal of internal limiting membrane in macular hole surgery: histology and outcome. American journal of ophthalmology. 2001;132(2):178-183.

16. Kumar A, Prakash G, Singh RP. Indocyanine green enhanced maculorhexis in macular hole surgery. Indian journal of ophthalmology. 2002;50(2):123-126.

17. Sheidow TG, Blinder KJ, Holekamp N, et al. Outcome results in macular hole surgery: an evaluation of internal limiting membrane peeling with and without indocyanine green. Ophthalmology. 2003;110(9):1697-1701. 
18. Gandorfer A, Haritoglou C, Gass CA, Ulbig MW, Kampik A. Indocyanine green-assisted peeling of the internal limiting membrane may cause retinal damage. American journal of ophthalmology. 2001;132(3):431-433.

19. Gass CA, Haritoglou C, Schaumberger M, Kampik A. Functional outcome of macular hole surgery with and without indocyanine green-assisted peeling of the internal limiting membrane. Graefe's archive for clinical and experimental ophthalmology = Albrecht von Graefes Archiv fur klinische und experimentelle Ophthalmologie. 2003;241(9):716-720.

20. Kanda S, Uemura A, Yamashita T, Kita H, Yamakiri K, Sakamoto T. Visual field defects after intravitreous administration of indocyanine green in macular hole surgery. Archives of ophthalmology (Chicago, Ill : 1960).

2004;122(10):1447-1451.

21. Haritoglou C, Gandorfer A, Gass CA, Schaumberger M, Ulbig MW, Kampik A. Indocyanine green-assisted peeling of the internal limiting membrane in macular hole surgery affects visual outcome: a clinicopathologic correlation. American journal of ophthalmology. 2002;134(6):836-841.

22. Ando F, Sasano K, Ohba N, Hirose H, Yasui O. Anatomic and visual outcomes after indocyanine green-assisted peeling of the retinal internal limiting membrane in idiopathic macular hole surgery. American journal of ophthalmology. 2004;137(4):609-614.

23. Posselt D, Rahman R, Smith M, Simcock PR. Visual outcomes following ICG assisted ILM peel for Macular Hole. Eye (London, England). 2005;19(3):279283.

24. Engelbrecht NE, Freeman J, Sternberg P, Jr., et al. Retinal pigment epithelial changes after macular hole surgery with indocyanine green-assisted internal limiting membrane peeling. American journal of ophthalmology. 2002;133(1):89-94.

25. Hirata A, Inomata Y, Kawaji T, Tanihara H. Persistent subretinal indocyanine green induces retinal pigment epithelium atrophy. American journal of ophthalmology. 2003;136(2):353-355.

26. Banker AS, Freeman WR, Kim JW, Munguia D, Azen SP. Vision-threatening complications of surgery for full-thickness macular holes. Vitrectomy for Macular Hole Study Group. Ophthalmology. 1997;104(9):1442-1452; discussion 1452-1443.

27. Haritoglou C, Schumann R, Reiniger I, et al. Evaluation of the internal limiting membrane after conventional peeling during macular hole surgery. Retina (Philadelphia, Pa). 2006;26(1):21-24.

28. Ben Simon GJ, Desatnik H, Alhalel A, Treister G, Moisseiev J. Retrospective analysis of vitrectomy with and without internal limiting membrane peeling for stage 3 and 4 macular hole. Ophthalmic surgery, lasers \& imaging : the official journal of the International Society for Imaging in the Eye. 2004;35(2):109-115.

29. Horio N, Horiguchi M. Effect on visual outcome after macular hole surgery when staining the internal limiting membrane with indocyanine green dye. Archives of ophthalmology (Chicago, Ill : 1960). 2004;122(7):992-996.

30. Brasil OM, Brasil OF. [Comparative analysis of macular hole surgery followed by internal limiting membrane removal with and without indocyanine green staining]. Arquivos brasileiros de oftalmologia. 2006;69(2):157-160.

31. Kumagai K, Furukawa M, Ogino N, Uemura A, Larson E. Long-term outcomes of internal limiting membrane peeling with and without indocyanine green in macular hole surgery. Retina (Philadelphia, Pa). 2006;26(6):613-617. 
32. $\mathrm{Wu} \mathrm{Y}, \mathrm{Zhu} \mathrm{W}, \mathrm{Xu} \mathrm{D}$, et al. Indocyanine green-assisted internal limiting membrane peeling in macular hole surgery: a meta-analysis. PloS one. 2012;7(11):e48405.

33. Ho JD, Tsai RJ, Chen SN, Chen HC. Cytotoxicity of indocyanine green on retinal pigment epithelium: implications for macular hole surgery. Archives of ophthalmology (Chicago, Ill : 1960). 2003;121(10):1423-1429.

34. Enaida H, Sakamoto T, Hisatomi T, Goto Y, Ishibashi T. Morphological and functional damage of the retina caused by intravitreous indocyanine green in rat eyes. Graefe's archive for clinical and experimental ophthalmology = Albrecht von Graefes Archiv fur klinische und experimentelle Ophthalmologie. 2002;240(3):209-213.

35. Ando F, Sasano K, Suzuki F, Ohba N. Indocyanine green-assisted ILM peeling in macular hole surgery revisited. American journal of ophthalmology. 2004;138(5):886-887.

36. Kusaka S, Oshita T, Ohji M, Tano Y. Reduction of the toxic effect of indocyanine green on retinal pigment epithelium during macular hole surgery. Retina (Philadelphia, Pa). 2003;23(5):733-734.

37. Facino M, Mochi B, Lai S, Terrile R. A simple way to prevent indocyanine green from entering the subretinal space during vitrectomy for retinal detachment due to myopic macular hole. European journal of ophthalmology. 2004;14(3):269-271.

38. Kodjikian L, Richter T, Halberstadt M, et al. Toxic effects of indocyanine green, infracyanine green, and trypan blue on the human retinal pigmented epithelium. Graefe's archive for clinical and experimental ophthalmology $=$ Albrecht von Graefes Archiv fur klinische und experimentelle Ophthalmologie. 2005;243(9):917-925.

39. Yonekawa Y, Abbey AM, Shah AR, Thomas BJ, Capone A, Jr. Endoilluminator phototoxic maculopathy associated with combined ICG-assisted epiretinal membrane and internal limiting membrane peeling. Clinical ophthalmology (Auckland, NZ). 2014;8:2501-2506.

40. Haritoglou C, Gandorfer A, Schaumberger M, Tadayoni R, Gandorfer A, Kampik A. Light-absorbing properties and osmolarity of indocyanine-green depending on concentration and solvent medium. Investigative ophthalmology \& visual science. 2003;44(6):2722-2729.

41. Gandorfer A, Haritoglou C, Gandorfer A, Kampik A. Retinal damage from indocyanine green in experimental macular surgery. Investigative ophthalmology \& visual science. 2003;44(1):316-323.

42. Sippy BD, Engelbrecht NE, Hubbard GB, et al. Indocyanine green effect on cultured human retinal pigment epithelial cells: implication for macular hole surgery. American journal of ophthalmology. 2001;132(3):433-435.

43. Ho JD, Tsai RJ, Chen SN, Chen HC. Toxic effect of indocyanine green on retinal pigment epithelium related to osmotic effects of the solvent. American journal of ophthalmology. 2003;135(2):258; author reply 259.

44. Penha FM, Maia M, Eid Farah M, et al. Effects of subretinal injections of indocyanine green, trypan blue, and glucose in rabbit eyes. Ophthalmology. 2007;114(5):899-908.

45. Sakamoto H, Yamanaka I, Kubota T, Ishibashi T. Indocyanine green-assisted peeling of the epiretinal membrane in proliferative vitreoretinopathy. Graefe's archive for clinical and experimental ophthalmology $=$ Albrecht von Graefes Archiv fur klinische und experimentelle Ophthalmologie. 2003;241(3):204-207. 
46. Kusaka S, Hayashi N, Ohji M, Hayashi A, Kamei M, Tano Y. Indocyanine green facilitates removal of epiretinal and internal limiting membranes in myopic eyes with retinal detachment. American journal of ophthalmology. 2001;131(3):388-390.

47. Mariotti C, Nicolai M, Donati S, Reibaldi M. Negative staining of the vitreous with the use of vital dyes. European journal of ophthalmology. 2018;28(1):117118.

48. Van De Moere A, Stalmans P. Anatomical and visual outcome of macular hole surgery with infracyanine green-assisted peeling of the internal limiting membrane, endodrainage, and silicone oil tamponade. American journal of ophthalmology. 2003;136(5):879-887.

49. La Heij EC, Dieudonne SC, Mooy CM, et al. Immunohistochemical analysis of the internal limiting membrane peeled with infracyanine green. American journal of ophthalmology. 2005;140(6):1123-1125.

50. Lanzetta P, Polito A, Del Borrello M, et al. Idiopathic macular hole surgery with low-concentration infracyanine green-assisted peeling of the internal limiting membrane. American journal of ophthalmology. 2006;142(5):771-776.

51. Garweg JG, Bergstein D, Windisch B, Koerner F, Halberstadt M. Recovery of visual field and acuity after removal of epiretinal and inner limiting membranes. The British journal of ophthalmology. 2008;92(2):220-224.

52. Feldman A, Zerbib J, Glacet-Bernard A, Haymann P, Soubrane G. Clinical evaluation of the use of infracyanine green staining for internal limiting membrane peeling in epimacular membrane surgery. European journal of ophthalmology. 2008;18(6):972-979.

53. Shimada H, Nakashizuka H, Hattori T, Mori R, Mizutani Y, Yuzawa M. Double staining with brilliant blue $\mathrm{G}$ and double peeling for epiretinal membranes. Ophthalmology. 2009;116(7):1370-1376.

54. Azuma K, Noda Y, Hirasawa K, Ueta T. BRILLIANT BLUE G-ASSISTED INTERNAL LIMITING MEMBRANE PEELING FOR MACULAR HOLE: A Systematic Review of Literature and Meta-Analysis. Retina (Philadelphia, Pa). 2016;36(5):851-858.

55. Mochizuki N, Yamamoto T, Enaida H, Ishibashi T, Yamashita H. Long-term outcomes of 3 surgical adjuvants used for internal limiting membrane peeling in idiopathic macular hole surgery. Japanese journal of ophthalmology. 2014;58(6):455-461.

56. Henrich PB, Priglinger SG, Haritoglou C, et al. Quantification of Contrast Recognizability during Brilliant Blue G- and Indocyanine Green-Assisted Chromovitrectomy. Investigative ophthalmology \& visual science. 2011;52(7):4345-4349.

57. Jindal A, Pathengay A, Mithal K, Chhablani J, Pappuru RR, Flynn HW. Macular toxicity following brilliant blue G-assisted macular hole surgery - a report of three cases. Nepalese journal of ophthalmology : a biannual peer-reviewed academic journal of the Nepal Ophthalmic Society : NEPJOPH. 2014;6(11):98101.

58. Schumann RG, Gandorfer A, Priglinger SG, Kampik A, Haritoglou C. Vital dyes for macular surgery: a comparative electron microscopy study of the internal limiting membrane. Retina (Philadelphia, Pa). 2009;29(5):669-676.

59. Chalam KV, Li W, Koushan K, Grover S, Balaiya S. Effect of distance and duration of illumination on retinal ganglion cells exposed to varying 
concentrations of brilliant blue green. Journal of clinical medicine research. 2015;7(7):517-524.

60. Martidis A, Duker JS, Greenberg PB, et al. Intravitreal triamcinolone for refractory diabetic macular edema. Ophthalmology. 2002;109(5):920-927.

61. Peyman GA, Cheema R, Conway MD, Fang T. Triamcinolone acetonide as an aid to visualization of the vitreous and the posterior hyaloid during pars plana vitrectomy. Retina (Philadelphia, Pa). 2000;20(5):554-555.

62. Sakamoto T, Miyazaki M, Hisatomi T, et al. Triamcinolone-assisted pars plana vitrectomy improves the surgical procedures and decreases the postoperative blood-ocular barrier breakdown. Graefe's archive for clinical and experimental ophthalmology $=$ Albrecht von Graefes Archiv fur klinische und experimentelle Ophthalmologie. 2002;240(6):423-429.

63. Fraser EA, Cheema RA, Roberts MA. Triamcinolone acetonide-assisted peeling of retinal internal limiting membrane for macular surgery. Retina (Philadelphia, Pa). 2003;23(6):883-884.

64. Couch SM, Bakri SJ. Use of triamcinolone during vitrectomy surgery to visualize membranes and vitreous. Clinical ophthalmology (Auckland, NZ). 2008;2(4):891-896.

65. Kumagai K, Furukawa M, Ogino N, Larson E, Uemura A. Long-term outcomes of macular hole surgery with triamcinolone acetonide-assisted internal limiting membrane peeling. Retina (Philadelphia, Pa). 2007;27(9):1249-1254.

66. Tsipursky MS, Heller MA, De Souza SA, et al. Comparative evaluation of no dye assistance, indocyanine green and triamcinolone acetonide for internal limiting membrane peeling during macular hole surgery. Retina (Philadelphia, Pa). 2013;33(6):1123-1131.

67. Nomoto H, Shiraga F, Yamaji H, et al. Macular hole surgery with triamcinolone acetonide-assisted internal limiting membrane peeling: one-year results. Retina (Philadelphia, Pa). 2008;28(3):427-432.

68. Ueno A, Enaida H, Hata Y, et al. Long-term clinical outcomes and therapeutic benefits of triamcinolone-assisted pars plana vitrectomy for proliferative vitreoretinopathy: a case study. European journal of ophthalmology. 2007;17(3):392-398.

69. Enaida H, Hata Y, Ueno A, et al. Possible benefits of triamcinolone-assisted pars plana vitrectomy for retinal diseases. Retina (Philadelphia, $\mathrm{Pa}$ ). 2003;23(6):764-770.

70. Takeuchi M, Katagiri Y, Usui M. Residual triamcinolone acetonide in the macular hole after vitrectomy. American journal of ophthalmology. 2003;136(6):1174-1176.

71. Yamauchi Y, Nakamura H, Hayakawa K, Sawaguchi S. Persistence of triamcinolone acetonide following macular hole surgery. Acta ophthalmologica Scandinavica. 2006;84(5):711-712.

72. Hikichi T, Furukawa Y, Ohtsuka H, et al. Improvement of visual acuity one-year after vitreous surgery in eyes with residual triamcinolone acetonide at the macular hole. American journal of ophthalmology. 2008;145(2):267-272.

73. Beer PM, Bakri SJ, Singh RJ, Liu W, Peters GB, 3rd, Miller M. Intraocular concentration and pharmacokinetics of triamcinolone acetonide after a single intravitreal injection. Ophthalmology. 2003;110(4):681-686.

74. Feron EJ, Veckeneer M, Parys-Van Ginderdeuren R, Van Lommel A, Melles GR, Stalmans P. Trypan blue staining of epiretinal membranes in proliferative 
vitreoretinopathy. Archives of ophthalmology (Chicago, Ill : 1960). 2002;120(2):141-144.

75. Perrier M, Sebag M. Trypan blue-assisted peeling of the internal limiting membrane during macular hole surgery. American journal of ophthalmology. 2003;135(6):903-905.

76. Teba FA, Mohr A, Eckardt C, et al. Trypan blue staining in vitreoretinal surgery. Ophthalmology. 2003;110(12):2409-2412.

77. Kwok AK, Lai TY, Li WW, Yew DT, Wong VW. Trypan blue- and indocyanine green-assisted epiretinal membrane surgery: clinical and histopathological studies. Eye (London, England). 2004;18(9):882-888.

78. Haritoglou C, Gandorfer A, Schaumberger M, et al. Trypan blue in macular pucker surgery: an evaluation of histology and functional outcome. Retina (Philadelphia, Pa). 2004;24(4):582-590.

79. Vote BJ, Russell MK, Joondeph BC. Trypan blue-assisted vitrectomy. Retina (Philadelphia, Pa). 2004;24(5):736-738.

80. Abdelkader EA, McBain VA, Anand M, Scott NW, Rehman Siddiqui MA, Lois $\mathrm{N}$. In vivo safety of trypan blue use in vitreoretinal surgery. Retina (Philadelphia, Pa). 2011;31(6):1122-1127.

81. Rodrigues EB, Meyer CH, Schmidt JC, Kroll P. Trypan blue stains the epiretinal membrane but not the internal limiting membrane. The British journal of ophthalmology. 2003;87(11):1431-1432.

82. Meyer CH, Rodrigues EB, Kroll P. Trypan blue has a high affinity to cellular structures such as epiretinal membrane. American journal of ophthalmology. 2004;137(1):207-208; author reply 208.

83. Jain S, Kishore K, Sharma YR. Progressive atrophy of retinal pigment epithelium after trypan-blue-assisted ILM peeling for macular hole surgery. Indian journal of ophthalmology. 2013;61(5):235-237.

84. Rishi P, Rishi E, Kothari A. Retinal pigment epithelial changes after trypan blue-assisted internal limiting membrane peeling for idiopathic macular hole. Oman journal of ophthalmology. 2011;4(1):37-38.

85. Li K, Wong D, Hiscott P, Stanga P, Groenewald C, McGalliard J. Trypan blue staining of internal limiting membrane and epiretinal membrane during vitrectomy: visual results and histopathological findings. The British journal of ophthalmology. 2003;87(2):216-219.

86. Mackenzie SE, Gandorfer A, Rohleder M, et al. Ultrastructure and retinal imaging of internal limiting membrane: a clinicopathologic correlation of trypan blue stain in macular hole surgery. Retina (Philadelphia, Pa). 2010;30(4):655661.

87. Haritoglou C, Eibl K, Schaumberger M, et al. Functional outcome after trypan blue-assisted vitrectomy for macular pucker: a prospective, randomized, comparative trial. American journal of ophthalmology. 2004;138(1):1-5.

88. Christensen UC, Kroyer K, Sander B, et al. Value of internal limiting membrane peeling in surgery for idiopathic macular hole stage 2 and 3: a randomised clinical trial. The British journal of ophthalmology. 2009;93(8):1005-1015.

89. Rodrigues EB, Penha FM, de Paula Fiod Costa E, et al. Ability of new vital dyes to stain intraocular membranes and tissues in ocular surgery. American journal of ophthalmology. 2010;149(2):265-277.

90. Haritoglou C, Yu A, Freyer W, et al. An evaluation of novel vital dyes for intraocular surgery. Investigative ophthalmology \& visual science. 2005;46(9):3315-3322. 
91. Haritoglou C, Tadayoni R, May CA, et al. Short-term in vivo evaluation of novel vital dyes for intraocular surgery. Retina (Philadelphia, $\mathrm{Pa}$ ). 2006;26(6):673-678.

92. Giansanti F, Schiavone N, Papucci L, et al. Safety testing of blue vital dyes using cell culture models. Journal of ocular pharmacology and therapeutics : the official journal of the Association for Ocular Pharmacology and Therapeutics. 2014;30(5):406-412.

93. Schuettauf F, Haritoglou C, May CA, et al. Administration of novel dyes for intraocular surgery: an in vivo toxicity animal study. Investigative ophthalmology \& visual science. 2006;47(8):3573-3578.

94. Jackson TL, Griffin L, Vote B, Hillenkamp J, Marshall J. An experimental method for testing novel retinal vital stains. Experimental eye research. 2005;81(4):446-454.

95. Penha FM, Pons M, Costa Ede P, et al. Effect of vital dyes on retinal pigmented epithelial cell viability and apoptosis: implications for chromovitrectomy. Ophthalmologica Journal international d'ophtalmologie International journal of ophthalmology Zeitschrift fur Augenheilkunde. 2013;230 Suppl 2:41-50.

96. Rodrigues EB, Penha FM, Farah ME, et al. Preclinical investigation of the retinal biocompatibility of six novel vital dyes for chromovitrectomy. Retina (Philadelphia, Pa). 2009;29(4):497-510.

97. Costa EF, Barros NM, Coppini LP, et al. Effects of light exposure, $\mathrm{pH}$, osmolarity, and solvent on the retinal pigment epithelial toxicity of vital dyes. American journal of ophthalmology. 2013;155(4):705-712, 712.e701.

98. Hata Y, Enaida H, Sassa Y, et al. Preclinical investigation of fluorometholone acetate as a potential new adjuvant during vitreous surgery. Graefe's archive for clinical and experimental ophthalmology = Albrecht von Graefes Archiv fur klinische und experimentelle Ophthalmologie. 2007;245(7):1019-1025.

99. Badaro E, Souza-Lima RA, Novais EA, et al. Investigation of new dyes for chromovitrectomy: preclinical biocompatibility of trisodium, orangell and methyl violet. International journal of retina and vitreous. 2015;1:1.

100. Liu Z, Meyer CH, Stanzel BV. Effect of novel vital dyes on outer blood-retina barrier function in cultured human retinal pigment epithelium. Ophthalmologica Journal international d'ophtalmologie International journal of ophthalmology Zeitschrift fur Augenheilkunde. 2013;230 Suppl 2:33-40.

101. Luke C, Luke M, Dietlein TS, et al. Retinal tolerance to dyes. The British journal of ophthalmology. 2005;89(9):1188-1191.

102. Luke C, Luke M, Sickel W, Schneider T. Effects of patent blue on human retinal function. Graefe's archive for clinical and experimental ophthalmology = Albrecht von Graefes Archiv fur klinische und experimentelle Ophthalmologie. 2006;244(9):1188-1190.

103. Mennel S, Thumann G, Peter S, Meyer CH, Kroll P. [Influence of vital dyes on the function of the outer blood-retinal barrier in vitro]. Klinische Monatsblatter fur Augenheilkunde. 2006;223(7):568-576.

104. Maia M, Penha F, Rodrigues EB, et al. Effects of subretinal injection of patent blue and trypan blue in rabbits. Current eye research. 2007;32(4):309-317.

105. Morales MC, Freire V, Asumendi A, et al. Comparative effects of six intraocular vital dyes on retinal pigment epithelial cells. Investigative ophthalmology \& visual science. 2010;51(11):6018-6029.

106. Luke M, Grisanti S, Luke J. The retinal biocompatibility of dyes in the ex vivo model of the isolated superfused vertebrate retina. Ophthalmologica Journal 
international d'ophtalmologie International journal of ophthalmology Zeitschrift fur Augenheilkunde. 2013;230 Suppl 2:21-26.

107. Haritoglou C, Kreutzer T, Tadayoni R, et al. Staining and peeling of the internal limiting membrane using a fluorescent dye (Rhodamine $6 \mathrm{G})$. The British journal of ophthalmology. 2008;92(9):1265-1268.

108. Balaiya S, Brar VS, Murthy RK, Chalam KV. Comparative in vitro safety analysis of dyes for chromovitrectomy: indocyanine green, brilliant blue green, bromophenol blue, and infracyanine green. Retina (Philadelphia, Pa). 2011;31(6):1128-1136.

109. Haritoglou C, Strauss R, Priglinger SG, Kreutzer T, Kampik A. Delineation of the vitreous and posterior hyaloid using bromophenol blue. Retina (Philadelphia, Pa). 2008;28(2):333-339.

110. Awad D, Wilinska J, Gousia D, et al. Toxicity and phototoxicity in human ARPE-19 retinal pigment epithelium cells of dyes commonly used in retinal surgery. European journal of ophthalmology. 2018:1120672118766446.

111. Haritoglou C, Schumann RG, Strauss R, Priglinger SG, Neubauer AS, Kampik A. Vitreoretinal surgery using bromphenol blue as a vital stain: evaluation of staining characteristics in humans. The British journal of ophthalmology. 2007;91(9):1125-1128.

112. Penha FM, Maia M, Farah ME, et al. Morphologic and clinical effects of subretinal injection of indocyanine green and infracyanine green in rabbits. Journal of ocular pharmacology and therapeutics : the official journal of the Association for Ocular Pharmacology and Therapeutics. 2008;24(1):52-61.

113. Schurmans A, Van Calster J, Stalmans P. Macular hole surgery with inner limiting membrane peeling, endodrainage, and heavy silicone oil tamponade. American journal of ophthalmology. 2009;147(3):495-500.

114. Furlani BA, Barroso L, Sousa-Martins D, et al. Lutein and zeaxanthin toxicity with and without brilliant blue in rabbits. Journal of ocular pharmacology and therapeutics : the official journal of the Association for Ocular Pharmacology and Therapeutics. 2014;30(7):559-566.

115. Casaroli-Marano RP, Sousa-Martins D, Martinez-Conesa EM, et al. Dye solutions based on lutein and zeaxanthin: in vitro and in vivo analysis of ocular toxicity profiles. Current eye research. 2015;40(7):707-718.

116. Badaro E, Furlani B, Prazeres J, et al. Soluble lutein in combination with brilliant blue as a new dye for chromovitrectomy. Graefe's archive for clinical and experimental ophthalmology = Albrecht von Graefes Archiv fur klinische und experimentelle Ophthalmologie. 2014;252(7):1071-1078.

117. Caiado RR, Peris CS, Lima-Filho AAS, et al. Retinal Toxicity of Acai Fruit (Euterpe Oleracea) Dye Concentrations in Rabbits: Basic Principles of a New Dye for Chromovitrectomy in Humans. Current eye research. 2017;42(8):11851193.

118. Chen J, Ferreira MA, Farah ME, et al. Posterior hyaloid detachment and internal limiting membrane peeling assisted by anthocyanins from acai fruit (Euterpe oleracea) and 10 other natural vital dyes: experimental study in cadaveric eyes. Retina (Philadelphia, Pa). 2013;33(1):89-96.

119. Tura A, Alt A, Haritoglou C, et al. Testing the effects of the dye acid violet-17 on retinal function for an intraocular application in vitreo-retinal surgery. Graefe's archive for clinical and experimental ophthalmology $=$ Albrecht von Graefes Archiv fur klinische und experimentelle Ophthalmologie. 2014;252(12):1927-1937. 
120. Tura A, Alt A, Luke J, et al. Biocompatibility of the vital dye Acid Violet-17 on retinal pigment epithelial cells. Clinical ophthalmology (Auckland, NZ). 2016;10:1435-1445.

121. Gerding H. Acid Violet 17: a New Dye for Chromovitrectomy? Klinische Monatsblatter fur Augenheilkunde. 2016;233(4):460-464.

122. Gerding H. Intraocular use of acid violet 17 at a concentration of $1.5 \mathrm{mg} / \mathrm{ml}$ is not safe. Graefe's archive for clinical and experimental ophthalmology = Albrecht von Graefes Archiv fur klinische und experimentelle Ophthalmologie. 2017;255(3):627-628.

123. Steel DH. Letter in response to letter: intraocular use of acid violet 17 at a concentration of $1.5 \mathrm{mg} / \mathrm{ml}$ is not safe : By Professor Heinrich Gerding, Department of Ophthalmology, Pallas Kliniken. Graefe's archive for clinical and experimental ophthalmology $=$ Albrecht von Graefes Archiv fur klinische und experimentelle Ophthalmologie. 2017;255(3):629.

124. Hurst J, Schnichels S, Spitzer MS, et al. Negative Effects of Acid Violet-17 and MBB Dual In Vitro on Different Ocular Cell Lines. Current eye research. 2017;42(8):1209-1214.

Figure Legends

Figure 1. US retina specialist ILM/ERM removal dye preference over time. The data, obtained from annual ASRS preference and trends surveys, demonstrates an increasing popularity of ICG for ILM and/or ERM peeling over the last decade, and a trends towards a decrease in the use of TA and TB. BBG, brilliant blue; ERM, epiretinal membrane; ICG, indocyanine green; ILM, internal limiting membrane; TA, triamcinolone acetonide; TB, trypan blue. The data for this graph was obtained and used in this manuscript with permission from the ASRS. ASRS, American Society of Retina Specialists; BBG, Brilliant Blue G; ERM, epiretinal membrane; ICG, indocyanine green; ILM, internal limiting membrane; TB, trypan blue; US, United States.

Figure 2: Indocyanine green staining of epiretinal membrane (ERM) and internal limiting membrane (ILM). In image plane A, the poor staining of ERM is demonstrated by the translucent ERM tissue visualized over the contrasting macular 
hole (white arrow). In contrast, in image B, the excellent staining of ILM is readily visualized (black arrow). Photographs courtesy of Dr. Caroline Baumal.

Figure 3. Brilliant Blue G (BBG) staining of the internal limiting membrane (ILM) in idiopathic macular hole repair. The blue staining of the ILM with BBG provides excellent contrast with the underlying neurosensory retina and surrounding ILM. Photograph courtesy of Dr. Thomas Ciulla. 
Table 3. Comparison of various dyes, their ability to stain transparent ocular tissues and safety concerns.

\begin{tabular}{|l|c|c|c|c|}
\hline Dye & $\begin{array}{c}\text { Staining } \\
\text { of ILM }\end{array}$ & $\begin{array}{c}\text { Staining of } \\
\text { ERM }\end{array}$ & $\begin{array}{c}\text { Staining of } \\
\text { vitreous }\end{array}$ & Safety concerns \\
\hline Indocyanine green & +++ & + & + & +++ \\
\hline Trypan Blue & + & +++ & + & + \\
\hline Triamcinolone Acetonide & + & + & +++ & + \\
\hline Brilliant Blue G & ++ & + & + & + \\
\hline
\end{tabular}

This table provides a comparison of various dyes in their ability to stain transparent ocular tissues and safety concerns. ILM, internal limiting membrane; ERM epiretinal membrane; +, minimal; ++, moderate; +++, maximal. 
Figure 1. US retina specialist ILM/ERM removal dye preference over time.

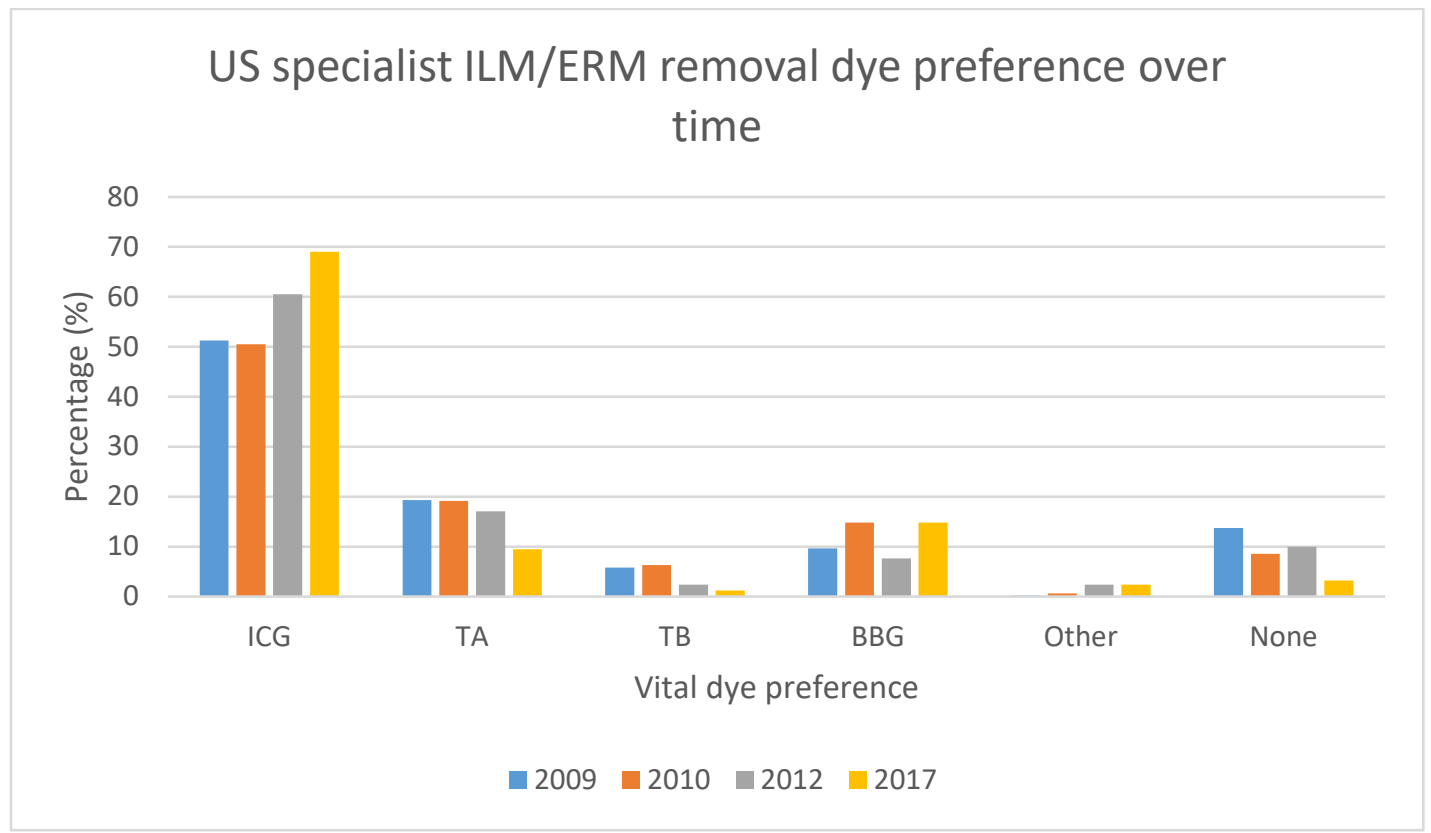


Figure 2: Indocyanine green staining of epiretinal membrane (ERM) and internal limiting membrane (ILM).

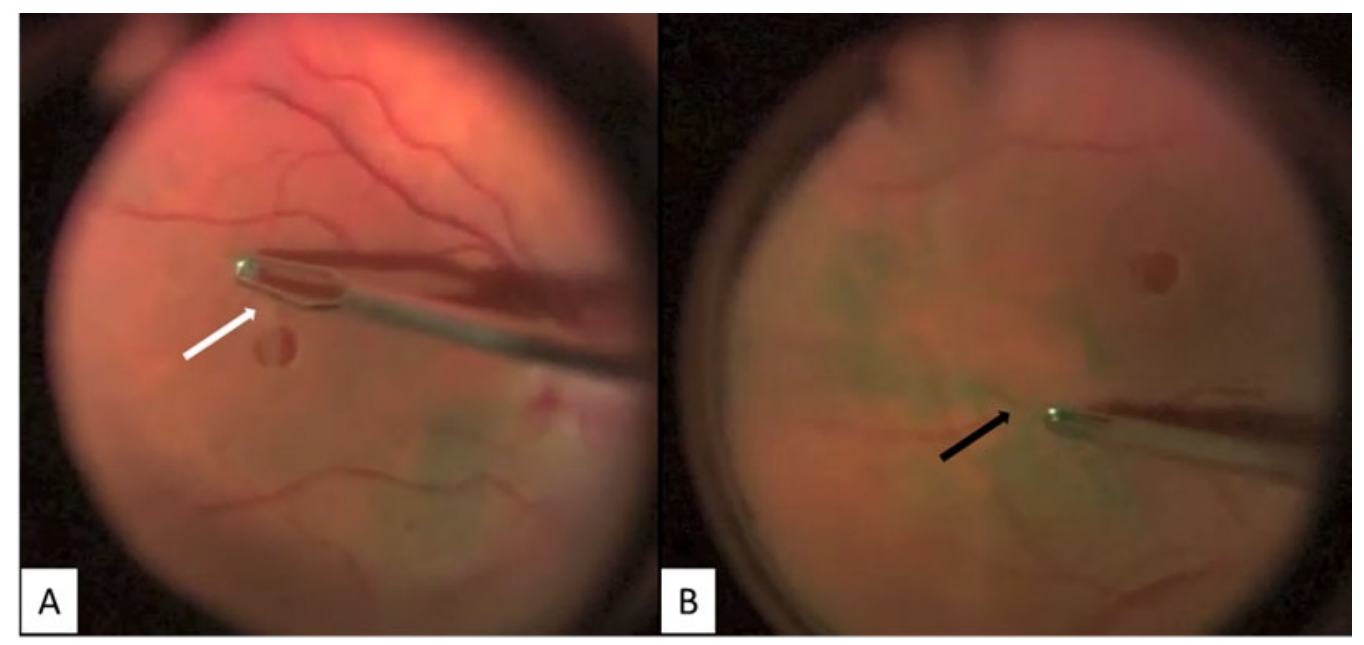


Figure 3. Brilliant Blue G (BBG) staining of the internal limiting membrane (ILM) in idiopathic macular hole repair.

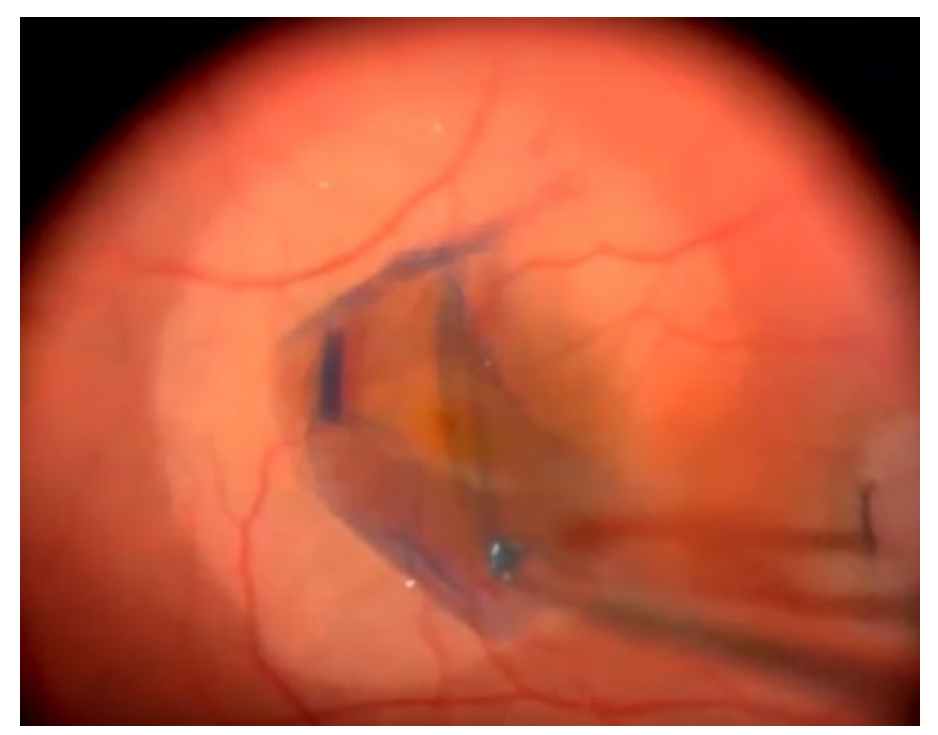

